\title{
Definiciones implícitas y unicidad en el programa neologicista
}

\author{
LUCAS ROSENBLATT \\ Consejo Nacional de Investigaciones Científicas y Técnicas \\ Universidad de Buenos Aires \\ 1_rosenblatt@hotmail.com
}

Resumen: En este trabajo presento un problema que afecta al programa neologicista que han defendido en varias ocasiones Crispin Wright y Bob Hale. En particular, argumento que Wright y Hale no han dado suficientes condiciones para separar las definiciones implícitas apropiadas como el principio de Hume de otras definiciones implícitas rivales como la aritmética de Peano de segundo orden. Sugiero, además, que esa tarea sólo puede realizarse adecuadamente si una de las condiciones propuestas es la condición de que toda definición implícita sea unívoca.

Palabras clave: neofregeanismo, teorema de Frege, aritmética de Peano, definiciones implícitas, unicidad

\begin{abstract}
In this paper I consider a problem affecting the Neologicist Program advocated on many occasions by Bob Hale and Crispin Wright. In particular, I argue that Hale and Wright have not given enough conditions to separate appropriate implicit definitions such as Hume's Principle from rival implicit definitions like Second-Order Peano Arithmetic. I also suggest that this task can only be performed adequately if one of the proposed conditions is that every implicit definition be univocal.
\end{abstract}

Key words: neo-Fregeanism, Frege's theorem, Peano arithmetic, implicit definitions, uniqueness

\section{Introducción}

En su clásico Frege's Conception of Numbers as Objects, Crispin Wright sugirió que es posible derivar los axiomas de Peano de la equivalencia conocida como principio de Hume mediante una lógica de segundo orden apropiada. Este resultado es el punto de partida de una posición filosófica que hoy se conoce como "neologicismo". ${ }^{1}$

Los neologicistas usualmente formulan el principio de Hume (que abreviaremos $\mathrm{PH}$ ) con un lenguaje de segundo orden (que incluye un predicado de identidad y un operador \#) de la siguiente forma:

$$
P H \quad \forall F \forall G(\#(F)=\#(G) \leftrightarrow F \approx G)
$$

${ }^{1}$ Algunos prefieren la denominación "neofregeanismo" o "abstraccionismo", ya que no consideran que los principios de los cuales se deriva la aritmética sean puramente lógicos. 
donde " $\approx$ " denota la relación de equivalencia numérica (definible por medio de una fórmula pura de segundo orden), "\#" representa una función que toma como argumento un concepto (o alguna entidad similar) y nos entrega como valor un objeto (un número), y " $F$ " y "G" son variables de segundo orden. Está claro que " $\#(F)$ " es un término singular que debe leerse como el número de las $F .^{2}$ Por lo tanto, $\mathrm{PH}$ establece una equivalencia entre la noción de identidad numérica y la noción de relación biunívoca (correspondencia uno-a-uno).

Hay en realidad diversas posiciones que pueden recibir el nombre "neologicismo". En su variante más conocida, la defendida por Bob Hale y el mismo Wright (de aquí en adelante $\mathrm{HyW}$ ), ${ }^{3}$ se establece que si colocamos a $\mathrm{PH}$ en conjunción con axiomas de un sistema lógico de segundo orden adecuado, es posible derivar (enunciados definicionalmente equivalentes a) los axiomas aritméticos usuales. Este resultado, conocido con el nombre de "teorema de Frege", va acompañado por una prueba de consistencia relativa que muestra que el sistema formado por una lógica apropiada de segundo orden y $P H$ (que llamaré de aquí en adelante $F A$, por "aritmética fregeana") es consistente si y sólo si la aritmética de Peano de segundo orden (que abreviaré $P A_{2}$ ) es consistente. $^{4}$

En manos de HyW estos resultados se convierten en el punto de partida de una especie de fundacionismo epistemológico y ontológico. El primer tipo de fundacionismo, que es el único que discutiré aquí, puede explicarse de la siguiente forma. Se pretende justificar nuestro conocimiento aritmético ${ }^{5}$ anclándolo en nuestro conocimiento de principios lógicos (de segundo orden) y en nuestro conocimiento de $\mathrm{PH}$. El procedimiento consiste en estipular la verdad de $\mathrm{PH}$ y hacer que este

${ }^{2}$ He decidido utilizar “\#(F)" en lugar de otras variantes disponibles en la bibliografía sobre el tema como " $N x: F x$ " o " $N x:[x: F x]$ ", aunque nada importante dependa de eso.

${ }^{3}$ El lector puede consultar los artículos en Hale y Wright 2001 para una defensa poderosa de diversos aspectos del programa neologicista.

${ }^{4}$ Este resultado fue conjeturado inicialmente en Wright 1983 y probado luego en diversos textos. Una de esas pruebas puede encontrarse en Boolos 1987. Uso la abreviatura $P A_{2}$ para el sistema que resulta de tomar $\mathcal{D}_{2}$ (véase la sección siguiente) y agregar los axiomas de Peano y el axioma de inducción (de segundo orden). Utilizaré la versión de $P A_{2}$ que cuenta con "0", "sucesor", "suma" y "multiplicación" como términos primitivos. Una presentación más detallada de $P A_{2}$ puede encontrarse en Smith 2007. Uso la abreviación $F A$ para el sistema que resulta de tomar $\mathcal{D}_{2}$ y agregar $\mathrm{PH}$ como axioma. Adviértase que $\mathcal{D}_{2}$ ya tiene un axioma de comprensión.

${ }^{5}$ Me referiré únicamente al caso de la aritmética, pero hay también versiones neologicistas de la teoría de conjuntos y el análisis. Véase, por ejemplo, Cook 2007.

Diánoia, vol. LIX, no. 72 (mayo de 2014). 
principio funcione como una definición implícita de \#. Suponiendo por el momento que las estipulaciones de este tipo no son problemáticas (más tarde tendremos tiempo de analizar esto con detenimiento) y son epistémicamente útiles, en el sentido de que nos otorgan cierta garantía para creer en la verdad de lo estipulado, la idea es ver si podemos adquirir una justificación a priori para creer en la verdad de las leyes aritméticas. Como, por el teorema de Frege, sabemos que la aritmética puede derivarse de $\mathrm{PH}$ y de principios lógicos de segundo orden, bajo la suposición de que los principios lógicos son justificables a priori y que $P H$ es un enunciado analítico (y por lo tanto también justificable a priori), se sigue que también la aritmética es justificable a priori.

Lo que pretendo hacer es discutir un problema que surge de una posición como la anterior. Para determinar si $P H$ (o $F A)^{6}$ logra otorgarle de manera adecuada algún valor semántico a \# y logra generar alguna garantía a priori para creer en la verdad de los enunciados que incluyen \#, es necesario tener alguna teoría general que nos indique cuándo una definición implícita es apropiada. Con esta intención, HyW ofrecen una serie de condiciones para separar las definiciones implícitas apropiadas como $P H$ de otras definiciones implícitas rivales como la aritmética de Peano de segundo orden. Argumentaré primero que las condiciones que HyW han ofrecido no son suficientes para ese propósito y, segundo, que es necesario introducir una condición adicional, la condición de que una definición implícita sea unívoca.

El resto del artículo se estructura del siguiente modo. En la sección 2 presentaré el aparato lógico necesario para comprender los aspectos formales de la posición neologicista que discutiré; en particular, introduciré la lógica subyacente que el neologicista necesita para probar el teorema de Frege. En la sección 3 consideraré las observaciones de HyW en torno a las condiciones de adecuación de las definiciones implícitas y sugeriré, por un lado, que éstas son insuficientes y, por otro, que la unicidad debe ser una de estas condiciones, algo que HyW no comparten. La sección 4 contiene algunas observaciones finales.

\footnotetext{
${ }^{6}$ Hablaré de manera indistinta de $P H$ o FA como definición implícita del operador numérico \#.
} 


\section{Lógica de segundo orden libre}

Sea $\mathcal{L}_{2}$ un lenguaje de segundo orden. ${ }^{7}$ Además de contar con variables individuales $\left(x_{1}, x_{2}, x_{3}, \ldots\right)$, conectivas y cuantificadores de primer orden $(\forall x$ y $\exists x)$, el vocabulario de $\mathcal{L}_{2}$ tiene variables de predicado $n$-ádicas $\left(X_{1}^{n}, X_{2}^{n}, X_{3}^{n}, \ldots\right)^{8}$ y cuantificadores de segundo orden $(\forall X \mathrm{y}$ $\exists X$ ). Por cuestiones de legibilidad, en ocasiones usaré las letras $F, G$, $H, R$, etc., como variables de predicado en lugar de $X_{i}^{n}$, y usaré $y, z, w$, etc., como variables individuales en lugar de $x_{i}$. Supondré también que $\mathcal{L}_{2}$ contiene el símbolo de función \#, cuya interpretación intuitiva es "el número de las...".

Es muy sencillo construir un sistema deductivo para $\mathcal{L}_{2}$ añadiendo algunos axiomas y/o reglas a un sistema deductivo usual de primer orden. Llamemos $\mathcal{D}_{2}$ a tal sistema. $\mathcal{D}_{2}$ tiene reglas y/o axiomas que gobiernan el comportamiento de los cuantificadores de segundo orden y un axioma-esquema de comprensión con la siguiente forma:

$$
\text { Comp } \quad \exists X^{n} \forall x_{1} \ldots \forall x_{n}\left(X^{n} x_{1} \ldots x_{n} \leftrightarrow \varphi\left(x_{1} \ldots x_{n}\right)\right)
$$

donde $\varphi$ no contiene ninguna variable libre además de $x_{1} \ldots x_{n}$. Este axioma afirma que toda fórmula $\varphi\left(x_{1} \ldots x_{n}\right)$ del lenguaje $\mathcal{L}_{2}$ que no tenga variables libres además de $x_{1} \ldots x_{n}$ determina una relación. Si la fórmula $\varphi\left(x_{1} \ldots x_{n}\right)$ contiene variables de orden superior ligadas, la instancia correspondiente se denomina "impredicativa"; en caso contrario, la instancia es "predicativa".

Ahora bien, de vuelta a $P H$, está claro que el lado derecho del bicondicional puede expresarse por medio de una fórmula pura de segundo orden, donde una fórmula pura de segundo orden es una fórmula de $\mathcal{L}_{2}$ que no contiene ninguna aparición del operador \#:

$F \approx G=_{\text {def }} \exists R(\forall x(F x \rightarrow \exists ! y(G y \wedge R x y)) \wedge \forall x(G x \rightarrow \exists ! y(F y \wedge R x y)))^{9}$

Algunas instancias de $F \approx G$ son teoremas de $\mathcal{D}_{2}$. Por ejemplo, un caso sencillo es aquel en que reemplazamos tanto $F x$ como $G x$ por $x \neq$

${ }^{7}$ El lector puede tomar como referencia el lenguaje L2K presentado en el capítulo 3 de Shapiro 1991. Usaré a menudo este lenguaje para ilustrar mis afirmaciones, aunque a veces simplificaré un poco la notación.

${ }^{8}$ Para los propósitos del neologicista, en realidad es suficiente que $\mathcal{L}_{2}$ contenga solamente variables de predicado monádicas y diádicas.

${ }^{9}$ Recordemos además que $\exists ! x \varphi(x)$ debe leerse como existe un único $x$ que satisface la condición $\varphi$, lo cual puede expresarse del siguiente modo: $\exists x(\varphi(x) \wedge$ $\forall y(\varphi(y) \rightarrow y=x))$. Por otra parte, tampoco es indispensable que "=" sea primitivo, ya que en segundo orden $x=y$ puede definirse como $\forall F(F x \leftrightarrow F y)$.

Diánoia, vol. LIX, no. 72 (mayo de 2014). 
$x$, que expresa el concepto de no ser idéntico a sí mismo, obteniendo así una fórmula que afirma la correspondencia biunívoca del concepto $x \neq x$ consigo mismo. Esto permite, a través de $P H$, obtener el siguiente teorema en $F A$ :

$$
\#(x \neq x)=\#(x \neq x)
$$

Como $\#(x \neq x)$ es un término singular que denota un objeto, podemos obtener también $\exists y(y=\#(x \neq x))$, que afirma que existe un objeto que es el número de las cosas no idénticas a sí mismas. Si, por otra parte, aceptamos la siguiente definición:

$$
0={ }_{\text {def }} \#(x \neq x)
$$

lo que se prueba es que existe el número 0 . El resto de los números naturales se define básicamente de manera análoga a partir del 0:

$$
\begin{array}{ll}
1 & ={ }_{\text {def }} \#(x=0) \\
2 & =_{\text {def }} \#(x=0 \vee x=1) \\
\ldots & \\
n+1 & ={ }_{\text {def }} \#(x=0 \vee \ldots \vee x=n)
\end{array}
$$

Así, queda probado que cualquier colección finita de números naturales y, por lo tanto, cualquier número natural que podamos expresar, existe.

El lector habrá notado que $\mathcal{D}_{2}$ y $P H$ se combinan maravillosamente. Para producir (algunos) teoremas aritméticos, $\mathrm{PH}$ se alimenta de las instancias lógicas de su lado derecho, y estas instancias dependen del sistema deductivo que se emplee (en este caso, $\mathcal{D}_{2}$ ), en particular, de las instancias de Comp que se admitan.

Un rasgo interesante señalado por varios críticos del proyecto neologicista (Shapiro y Weir 2007; Potter y Smiley 2001; Rumfit 2003) es que la lógica subyacente del sistema de segundo orden empleado para probar el teorema de Frege es (debe ser) una lógica libre. La razón es que $P H$, qua definición implícita del operador numérico, debe funcionar como garante de la referencialidad de los términos singulares de la forma $\# \varphi$. Es esencial para que $P H$ cumpla el papel que el neologicista pretende asignarle que su verdad sea la responsable de la referencialidad de estos términos. Sin embargo, en la lógica clásica la suposición de que los términos singulares siempre tienen referencia trivializa las pruebas de las fórmulas que establecen la existencia de los números naturales. Podríamos probar, por ejemplo,

$$
\exists y(y=\#(x \neq x))
$$


del modo siguiente:

$$
\begin{aligned}
& {[1] \quad \forall x(x=x)} \\
& {[2] \quad \#(x \neq x)=\#(x \neq x)} \\
& {[3] \quad \exists y(y=\#(x \neq x))}
\end{aligned}
$$

En esta miniprueba de $\exists y(y=\#(x \neq x))$ no interviene de ninguna forma $P H$. La introducción de la identidad numérica del paso [2] sólo depende de $\forall x(x=x)$ junto con el hecho de que podemos instanciar el cuantificador universal con cualquier término singular y el hecho de que $\#(x \neq x)$ es un término singular de $\mathfrak{L}_{2}$. Por su parte, el paso de [2] a [3] sólo depende de que sea posible introducir el cuantificador existencial irrestrictamente. Para que las pruebas no sean triviales (es decir, para que sólo usando $P H$ sea posible derivar verdades aritméticas como la anterior) el neologicista debe restringir de alguna forma las reglas que gobiernan el comportamiento de los cuantificadores. Como tanto la instanciación irrestricta del cuantificador universal como la generalización irrestricta del cuantificador existencial son reglas inválidas en ciertas lógicas libres, la sugerencia obvia es emplear una lógica de este tipo. Aquí consideraremos sólo dos.

Un camino incorrecto sería modificar $\mathcal{D}_{2}$ haciendo que en lugar de las reglas usuales $\forall$-elim y $\exists$-intro para los cuantificadores, tengamos:

$$
\begin{aligned}
& \forall \text {-elim } \operatorname{lis}_{\text {pos }}: \forall x \varphi(x), E(t) / \varphi(t) \\
& \exists \text {-intro }_{\text {pos }}: \varphi(t), E(t) / \exists x \varphi(x)
\end{aligned}
$$

donde $E(t)=_{\text {def }} \exists x(x=t$ ) (hay otras lecturas posibles de $E(t)$ que no consideraré).

Llamemos $\mathcal{D} \mathfrak{L}_{2 p o s}$ (por lógica libre positiva de segundo orden) al sistema que resulta de reemplazar $\forall$-elim y $\exists$-intro en $\mathcal{D}_{2}$ por estas dos reglas. ${ }^{10}$ La dificultad con $\mathcal{D} \mathfrak{L}_{2 \text { pos }}$ es que las reglas modificadas no validan el paso de [2] a [3] y ese paso también es necesario en las pruebas legítimas de existencia numérica, esto es, en las pruebas que apelan a $P H$. Para obtener, por ejemplo, $\exists y(y=\#(x \neq x))$ a partir de $\#(x \neq x)=\#(x \neq x)$ (lo cual a su vez fue obtenido de $x \neq x \approx x \neq x$

${ }^{10}$ En las presentaciones usuales de sistemas de deducción natural para lógicas libres, por ejemplo en Nolt 2010 y en Bencivenga 1986, también se reformulan $\forall$-intro y $\exists$-elim, pero como las restricciones importantes conciernen a $\forall$-elim y $\exists$ intro, me concentraré exclusivamente en estas últimas.

Diánoia, vol. LIX, no. 72 (mayo de 2014). 
vía $P H)$, necesitaríamos la premisa extra $E(\#(x \neq x))$. Sin embargo, nada nos garantiza que esto último se cumpla, lo cual sugiere que el neologicista no puede usar esta versión de la lógica libre.

Hay también otras razones que le impiden al neologicista emplear esta lógica. La manera más frecuente de interpretar una lógica de segundo orden libre positiva es por medio de una estructura $\left\langle d_{I}, d_{O}, I\right\rangle$, donde $d_{I}$ es el dominio interno, $d_{O}$ es el dominio externo e $I$ es la función de interpretación habitual. Estipulamos que $d_{I} \cap d_{O}=\varnothing,{ }^{11}$ que $d_{I}$ puede ser vacío, ${ }^{12}$ pero que $d_{I} \cup d_{O} \neq \varnothing$ (esto es, $d_{O}$ debe tener al menos un elemento). La idea detrás de la distinción entre el dominio interno y el externo es que, para no introducir complicaciones excesivas en la interpretación de los términos singulares no referenciales, se les asigna un elemento del dominio externo. ${ }^{13}$ Los cuantificadores de primer orden recorren solamente $d_{I}$, mientras que los cuantificadores de segundo orden recorren $P\left(d_{I} \cup d_{O}\right)$, siendo $P$ la operación potencia. Con respecto a la función $I$, si $t$ es un término singular y $R_{n}$ una constante de predicado $n$-ádica, $I(t) \in d_{I} \cup d_{O}$, e $I\left(R_{n}\right) \subseteq\left(d_{I} \cup d_{O}\right)^{n}$. Si $I(t) \in d_{O}$ (con lo cual $I(t) \notin d_{I}$ ), decimos que $t$ es no referencial o vacío. Shapiro y Weir (2007) muestran que en este tipo de semántica hay modelos con $d_{I}$ vacío (y también con $d_{I}$ finito) donde $P H$ es satisfacible. Esto es inaceptable dado que $P H$ debería ser verdadero en un modelo sólo si el dominio de ese modelo es infinito.

Un camino más viable que adoptar $\mathcal{D} \mathfrak{L}_{2 p o s}$ es adoptar $\mathcal{D} \mathfrak{L}_{2 \text { neg }}$ (por lógica libre negativa de segundo orden). ${ }^{14}$ Este sistema resulta de debilitar las reglas de los cuantificadores de esta forma:

${ }^{11}$ En algunas presentaciones se estipula, en cambio, que $d_{I} \subseteq d_{O}$. Véase de nuevo Nolt 2010 y Bencivenga 1986.

${ }^{12}$ El lector puede consultar Hale y Wright 2001, pp. 235-236, para una aceptación explícita de que el dominio puede ser vacío. De todas formas, adviértase que no debemos dar por sentado que, por ser libre, la lógica en cuestión permite modelos con el dominio vacío. Hay lógicas libres cuya única diferencia con la lógica clásica es la presencia de términos singulares no referenciales. Las teorías lógicas que, además, permiten dominios vacíos, suelen llamarse "lógicas inclusivas". Según parece, la lógica adoptada por HyW es inclusiva. Si quisiéramos que la lógica fuera libre pero no inclusiva, bastaría agregar $\exists x E x$ como axioma al sistema.

${ }^{13}$ Esto tiene consecuencias lógicas deseables (la ley de identidad $\forall x(x=x)$ es irrestrictamente válida; $t=t$ es verdadera aun cuando $t$ sea no referencial), pero también consecuencias conceptuales posiblemente indeseables (¿cuál es el estatus ontológico de los elementos del dominio externo?).

${ }^{14}$ La distinción entre lógica libre positiva y negativa puede encontrarse, por ejemplo, en Priest 2008. 


$$
\begin{array}{ll}
\forall \text {-elim } \text { neg }_{\text {ing }}: & \forall x \varphi(x), \psi(a) \text { (atómica) } / \varphi(a) \\
\exists \text {-intro }_{\text {neg }}: & \varphi(a), \psi(a) \text { (atómica) } / \exists x \varphi(x)^{15}
\end{array}
$$

Esta formulación tiene varias virtudes. Además de rescatar la idea de HyW de que una condición suficiente para que un término singular tenga referencia es su presencia en un enunciado atómico verdadero, estas reglas validan la prueba que utiliza el lado derecho de $\mathrm{PH}$ para probar $\exists y(y=\#(x \neq x)$ ) (y el resto de los enunciados de existencia numérica), invalidando la prueba trivial que parte de $\forall x(x=x) .{ }^{16}$ Además, ya no será posible (por cómo estarán construidos los modelos) encontrar dominios vacíos o finitos en los cuales $\mathrm{PH}$ sea verdadero. ${ }^{17}$

\section{Las definiciones implícitas y sus condiciones de adecuación}

Está claro que no toda definición implícita es adecuada. Para mostrar que $F A$ es una definición implícita adecuada del operador numérico \#, el neologicista debe convencernos de que satisface una serie de condiciones que toda definición implícita debe satisfacer. Resulta sorprendente que esta cuestión sea bastante difícil de resolver. Hay muchos enunciados propuestos como definiciones implícitas que no logran conferirle significado a los términos que los componen. Los principios que gobiernan la conectiva tonk son un ejemplo de eso, pero aun cuando no haya un paralelo claro entre la forma en que suele definirse tonk y la forma en que se pretende definir $\#,{ }^{18}$ el neologicista necesita ofrecer una lista de criterios para determinar si su definición de \# es legítima, y esa lista debe separar los principios aceptables como $\mathrm{PH}$ de los

\footnotetext{
${ }^{15}$ Sigo aquí la presentación de McFarlane 2009.

${ }^{16}$ El defecto de $\mathcal{D} \mathfrak{L}_{2 \text { neg }}$, si es en verdad un defecto, es que habrá instancias de $t=t$ (aquellas en las que $t$ carece de referencia) que son falsas. Por lo tanto, la ley de identidad no vale irrestrictamente.

${ }^{17}$ Sin embargo, Shapiro y Weir 2007 señalan que con este tipo de lógica libre el principio de Hume deja de ser epistemológicamente inocente. Más adelante (en la sección 3) veremos que el hecho de que $F A$ tenga una lógica subyacente libre, mientras que $P A_{2}$ no, desempeña un papel importante al considerar la condición de unicidad.

${ }^{18} \mathrm{El}$ problema aquí es que tonk suele definirse por medio de reglas de introducción y eliminación en un sistema de deducción natural, mientras que \# suele definirse por medio del axioma $P H$. De cualquier forma, el paralelismo puede hacerse patente formulando $\mathrm{PH}$ de forma esquemática y por medio de un par de reglas:
}

$$
\begin{array}{ll}
\#(\varphi)=\#(\psi) / \varphi \approx \psi & {[\# \text {-elim }]} \\
\varphi \approx \psi / \#(\varphi)=\#(\psi) & {[\# \text {-intro }]}
\end{array}
$$

Diánoia, vol. LIX, no. 72 (mayo de 2014). 
principios inaceptables como la inconsistente ley básica $V$, pero también de las definiciones implícitas rivales como los axiomas de la aritmética de Peano de segundo orden $P A_{2} \cdot{ }^{19}$

Hasta el momento he hablado en términos muy generales de $P H$ como una definición implícita. Sin embargo, en realidad $P H$ es un tipo particular de definición implícita: un principio de abstracción. ${ }^{20}$ Se suele llamar principio de abstracción a todo enunciado de la forma:

$$
\S(\alpha)=\S(\beta) \leftrightarrow E q(\alpha, \beta)
$$

donde " $\alpha$ " y " $\beta$ " son variables que deben sustituirse por entidades de cierta clase, " $\S$ " es una función unaria que ubica entidades del tipo de las que pueden ser los valores de $\alpha$ y $\beta$ sobre objetos y $E q$ es una relación de equivalencia (es decir, una relación reflexiva, transitiva y simétrica) para este mismo tipo de entidad. El problema más inmediato que nos compete es que no todo principio de abstracción es aceptable (esto vale para las definiciones implícitas en general). Algunos son directamente inconsistentes, mientras que otros generan dificultades un poco más sutiles. Por ejemplo, podemos hacer referencia nuevamente al principio de abstracción más conocido de la historia de la filosofía, la ley básica $V$ :

$$
\text { Ley } V \quad \forall F \forall G(\$ F=\$ G \leftrightarrow \forall x(F x \leftrightarrow G x))
$$

donde el operador "\$" toma un concepto como argumento y nos da su extensión como valor.

Si adoptamos un axioma de comprensión irrestricto, ley $V$ es inconsistente, a pesar de tener la misma forma que $P H$, de modo que no puede ser la mera forma de $P H$ lo que le confiere presuntas propiedades semánticas y epistémicas deseables. Tiene que haber algo más en juego. Algo similar sucede en el caso de las conectivas lógicas. Si

${ }^{19}$ El tipo de criterios que el neologicista debe ofrecer es materia de discusión. Aquí consideraré el problema del modo en que lo han planteado HyW, pero es posible adoptar un enfoque más formal que el de estos autores. En particular, varios partidarios y detractores del programa neologicista han buscado ofrecer criterios únicamente para principios de abstracción, y no para definiciones implícitas en general.

${ }^{20}$ Esto no es tan inmediato. El lado derecho de $\mathrm{PH}$ es una relación de equivalencia sólo si lo escribimos como $F \approx G$, pero ésta es una fórmula de $\mathcal{L}_{2}$ sólo en caso de que permitamos que los predicados de segundo orden aparezcan en la posición de sujeto. De cualquier forma, aquí supondré sin reparos que es posible entender $F A$ y otros enunciados semejantes como principios de abstracción. 
entendemos las reglas usuales de introducción y eliminación de la conjunción, disyunción, etc., como definiciones implícitas del significado de las correspondientes conectivas, tenemos el famoso problema suscitado por la conectiva tonk. Esta conectiva es a la conjunción y a la disyunción lo que ley $V$ es a $P H$ : un mal acompañante.

Curiosamente, HyW han optado por ofrecer una respuesta al problema de la mala compañía (que, en el marco del proyecto neologicista, se plantea exclusivamente para principios de abstracción) mediante criterios de adecuación para las definiciones implícitas en general. ${ }^{21}$ Es común pedir que una definición implícita satisfaga la condición de existencia, esto es, que exista al menos un concepto o función que satisfaga la definición, ${ }^{22}$ y la condición de unicidad, a saber, que exista a lo sumo un concepto o función que satisfaga la definición. En estos trabajos HyW van un poco más lejos y proponen cinco condiciones que una definición implícita debe satisfacer para ser aceptable: consistencia, conservatividad, generalidad, armonía y no arrogancia. Nótese que la unicidad no figura entre las condiciones.

No es necesario caracterizar la condición de consistencia. Ofreceré una caracterización informal del resto de las condiciones. Decimos que una definición es conservativa con respecto a una teoría $\tau$ si añadir la definición a $\tau$ no implica nuevos teoremas sobre la ontología de $\tau$. Aunque es difícil de caracterizar, la condición de generalidad consiste básicamente en la idea de que una expresión está apropiadamente definida si puede figurar en cualquier tipo de contexto permisible para su categoría sintáctica. Esto tiene que ver, por supuesto, con el problema de Julio César, que no tenemos tiempo de analizar aquí. Por su parte, la armonía puede entenderse como una generalización de la idea - usualmente aplicable a expresiones lógicas - de que debe haber una relación de cierto tipo entre las reglas de introducción y eliminación. En particular, se requiere que las consecuencias más fuertes obtenibles por medio de una regla de eliminación sean exactamente aquellas que permiten la aplicación de una regla de introducción. ${ }^{23}$

Las condiciones cumplen con la función de descartar otras definiciones implícitas que son, en algún sentido relevante, defectuosas. Por

${ }^{21}$ Esto se lleva a cabo fundamentalmente en Hale y Wright 2000 y 2009.

${ }^{22}$ Aquí la condición de existencia puede entenderse en términos de la condición de consistencia, ya que diremos que el concepto definido existe si es consistente. Lo opuesto de esta afirmación, que no tenemos la necesidad de considerar aquí, es, en mi opinión, más controvertido.

${ }^{23}$ Una caracterización un poco más completa de cada una de las condiciones puede encontrarse en Hale y Wright 2000 y 2009.

Diánoia, vol. LIX, no. 72 (mayo de 2014). 
ejemplo, la condición de consistencia descarta la ley $V$, y la condición de conservatividad descarta el principio de paridad. ${ }^{24}$ No me detendré a analizar de qué forma cada una de las condiciones es útil para descartar otros principios, pero sí me interesa considerar una condición que no he discutido hasta ahora, lo que HyW llaman "no arrogancia". La no arrogancia se utiliza para separar a $F A$ de su principal rival en este contexto, $\mathrm{PA}_{2}$. Para comprender a qué se refieren HyW con "no arrogancia" es útil leer el siguiente pasaje:

Llamemos arrogante a cualquier estipulación de una oración "\#f", cuya verdad [...] no puede ser afirmada justificadamente sin un trabajo epistémico colateral a posteriori. Entonces, la conexión tradicional entre las definiciones implícitas y lo a priori requiere que al menos algunas estipulaciones no sean arrogantes. ¿Cómo podemos circunscribir las arrogantes de modo tal que las separemos de las otras? (Hale y Wright 2000, p. 297) ${ }^{25}$

Como respuesta a esta pregunta, HyW conectan explícitamente la no arrogancia con el carácter esencialmente condicional de las estipulaciones admisibles. Consideremos los enunciados estipulativos Jack y CJack:

Jack: Jack el destripador es el perpetrador de estos crímenes.

CJack: Si alguien es el único perpetrador de estos crímenes, es Jack el destripador.

El primer enunciado, Jack, presupone "arrogantemente" que hay un único perpetrador de los crímenes, mientras que CJack evita esa presuposición gracias a su carácter condicional. La condicionalización funciona como un instrumento para evitar la presuposición de que hay sólo un objeto que satisface la condición. ${ }^{26}$

¿Qué ocurre con $F A$ ? ¿Es $F A$ una definición arrogante? La misma pregunta debe plantearse para los axiomas de $P A_{2}$. En el enfoque adoptado por HyW, $P H$ es, en algún sentido interesante, mejor que $P A_{2}$ sólo si existe alguna condición satisfecha por $F A$ pero no por $P A_{2}$. La condición crucial para HyW es la condición de no arrogancia y plantean

${ }^{24}$ Véase, por ejemplo, Wright 2001c [1997].

${ }^{25}$ Esta traducción y todas las restantes son de mi autoría. El símbolo \# no debe entenderse aquí como el operador numérico, sino como el contexto, sea éste cual fuere, en el que aparece la expresión $f$.

${ }^{26}$ McFarlane 2009 (pp. 454-455) ha sugerido que este ejemplo es inadecuado. Hale y Wright 2009 (p. 467) aceptan lo anterior, pero creen que hay otros ejemplos para los cuales necesitamos la no arrogancia. 
que $F A$ cumple con la condición de no arrogancia gracias al carácter (bi)condicional de $P H$, mientras que $P A_{2}$, al ser una estipulación directa no condicional del valor semántico de "0", "sucesor", "suma" y "multiplicación", no lo hace.

Por mi parte, considero que el neologicista está en una posición desventajosa. Si el concepto de no arrogancia se agota en la presencia de un condicional, el argumento del neologicista a favor de $F A$ es demasiado débil porque no descarta ciertas versiones condicionales de $P A_{2}$. Si el concepto de no arrogancia no se agota en la presencia de un condicional, el neologicista debe dar una explicación más clara de esa condición.

En MacFarlane 2009 se señala que si lo único que separa a $F A$ de $P A_{2}$ es la arrogancia de esta última teoría, y que si lo que evita la arrogancia de $F A$ es el carácter (bi)condicional de $P H$, lo único que el defensor de $P A_{2}$ debe hacer para garantizar la no arrogancia de su teoría es condicionalizarla del siguiente modo:

$$
\text { MF } \quad \forall x(x=x) \leftrightarrow \mathrm{PA}_{2}{ }^{27}
$$

Esto implica que las condiciones que se han propuesto hasta el momento son demasiado débiles porque clasifican como aceptables ciertas definiciones implícitas que el neologicista quiere eliminar. ¿Qué objeción puede tener el neologicista contra $M F$ ? Como bien señala McFarlane, no puede decir que los miembros del bicondicional son muy disparejos, que uno es lógico y ontológicamente inocente, mientras que el otro es aritmético y ontológicamente culpable: lo mismo parece suceder con $P H$.

Otra respuesta consiste en sugerir que la diferencia fundamental entre $M F$ y $P H$ es que la verdad de $M F$ es equivalente a la verdad de su lado derecho, pero que esto no ocurre con $P H$. En otras palabras, la no arrogancia no se agota en tener forma condicional, sino que el contenido de los enunciados que forman parte del condicional también importa. Aunque esta idea parece adecuada, HyW nos deben una explicación más compleja de la no arrogancia de $P H$ (y de la arrogancia de $M F$ y otros bicondicionales similares).

HyW también sugieren tímidamente (2009, pp. 466-467) que no está claro hasta qué punto $P A_{2}$ cumple con las condiciones de generalidad y armonía. No me interesa demasiado analizar cuidadosamente esta cuestión aquí. Todo depende, por supuesto, de cómo se caractericen rigurosamente estas dos condiciones. Si por "generalidad" entendemos que la definición es suficiente para identificar a 0 como el referente

${ }^{27}$ Aquí $\mathrm{PA}_{2}$ es la conjunción de todos los axiomas de $P A_{2}$, y cualquier verdad lógica del lado izquierdo serviría.

Diánoia, vol. LIX, no. 72 (mayo de 2014). 
de "0", a la función sucesor como el valor semántico del símbolo de función "sucesor", etc., entonces $P A_{2}$ no es lo suficientemente general, dado que define cualquier omega-secuencia, no solamente la que se pretende definir. De entrada, en ese caso, es difícil ver por qué, por ejemplo, un estructuralista que defienda $P A_{2}$ se comprometería con una condición tan fuerte de generalidad. En cambio, si por "generalidad" entendemos que las condiciones de verdad de la expresión deben estar determinadas para un rango suficientemente amplio de contextos, como parecen sugerir HyW al caracterizar esta condición, no hay razones para pensar que $P A_{2}$ no cumple con ella.

Algo similar podemos decir de la condición de armonía. Si por "armonía" entendemos algo muy parecido a la condición usualmente requerida para las conectivas lógicas, entonces esta condición ni siquiera puede aplicarse apropiadamente a las formulaciones usuales de $P A_{2}$. En cambio, si por "armonía" entendemos que las distintas definiciones implícitas (parciales) de una expresión deben trabajar conjuntamente de un modo razonable, entonces tampoco hay razones para pensar que $P A_{2}$ no cumple con esa condición, algo que HyW no tienen problemas en admitir.

Por otra parte, una objeción más fina que la de McFarlane y más cercana al espíritu del ejemplo propuesto por HyW es que el defensor de $P A_{2}$ también puede garantizar la no arrogancia de su teoría condicionalizándola por medio de un "condicional de Carnap". Esta estrategia es común en las teorías empíricas, donde también solemos decir que el significado de los términos teóricos queda definido implícitamente. Por ejemplo, sea $\tau$ (neutrino) una teoría que habla sobre los neutrinos. En ese caso, el valor semántico de "neutrino" está dado por la verdad de (por lo menos algunos de) los enunciados que forman esa teoría. En una terminología más esquemática, diríamos que si se estipula que la teoría $\tau(\mathrm{f})$ es verdadera, se sigue inmediatamente que el término " $\mathrm{f}$ " tiene cierto valor semántico. La teoría $\tau(\mathrm{f})$ funciona como una definición implícita del término "f". Sin embargo, obsérvese que, si es la verdad de $\tau(\mathrm{f})$ lo que le otorga a "f " el valor semántico que posee, como $\tau(\mathrm{f})$ es una teoría empírica refutable, surge la pregunta siguiente: ¿qué ocurre con el valor semántico de "f" si las evidencias empíricas muestran la falsedad de $\tau(\mathrm{f})$ ? El defensor de las definiciones implícitas estaría obligado a decir que el valor semántico de "f " ha cambiado o que "f" nunca tuvo valor semántico. Ninguna de estas respuestas suena del todo convincente. La falsedad de $\tau$ (flogisto), por ejemplo, no debería llevarnos a decir que "flogisto" no tiene valor semántico alguno o que su valor semántico no es el que creíamos que era. 
Si $\exists X \tau(X)$ es la oración de Ramsey de $\tau(\mathrm{f}),{ }^{28}$ decimos que el nuevo término, en este caso " $\mathrm{f}$ ", se introduce por medio del "condicional de Carnap":

$$
\exists X \tau(X) \rightarrow \tau(\mathrm{f})
$$

Si la oración de Ramsey capta adecuadamente todo el contenido de $\tau(\mathrm{f})$, este condicional debe leerse como una mera convención que indica de qué modo debe usarse el nuevo término "f". Por lo tanto, es un enunciado que puede sostenerse de manera a priori (al menos en algún sentido sustantivo de "a priori"). En palabras de HyW:

Este condicional expresa, en efecto, una convención para el uso del nuevo término "f". Al estar [el condicional] completamente despojado de contenido empírico, puede estipularse, o ser declarado verdadero a priori, sin que esto vaya en desmedro de la disconfirmabilidad empírica de la teoría. (Hale y Wright 2000, p. 309)

Entonces, en sentido estricto la teoría es la conjunción formada por el condicional carnapiano $\exists X \tau(X) \rightarrow \tau(\mathrm{f})$ y la oración de Ramsey $\exists X \tau(X)$, es decir:

$$
\exists X \tau(X) \wedge \exists X \tau(X) \rightarrow \tau(\mathrm{f})
$$

Pero el enunciado que realiza el trabajo estipulativo o definicional es el condicional carnapiano, lo cual impide que la estipulación sea arrogante.

Como la apelación al condicional carnapiano parece funcionar bien en el caso de las teorías empíricas, surge la tentación de extender esta estrategia al caso de las teorías lógicas y al de la aritmética. En el caso de las teorías lógicas, Boghossian 2003 ha sostenido que el empleo del condicional carnapiano lleva a algo parecido a un círculo vicioso o a una regresión infinita. Es fácil ver por qué. Supongamos que la expresión lógica cuyo valor semántico queremos fijar por medio del condicional carnapiano es el condicional material (o el cuantificador existencial).

\footnotetext{
${ }^{28}$ Suponiendo, para simplificar las cosas, que "f" es el único término que se introduce por medio de una teoría $\tau(\mathrm{f})$, la oración de Ramsey de esa teoría se obtiene poniendo en una conjunción todos los enunciados de dicha teoría, reemplazando todas las apariciones de "f " por una variable adecuada en la conjunción resultante y anteponiendo a este enunciado un cuantificador existencial apropiado. Si se define más de un término, como ocurre, por ejemplo, en la versión $P A_{2}$ que estamos usando, donde tenemos "cero", "sucesor", "suma" y "multiplicación", la cuantificación existencial es múltiple y puede haber más de un tipo de variable.
}

Diánoia, vol. LIX, no. 72 (mayo de 2014). 
Dado que la única manera de expresar un condicional de este tipo es usando un condicional material (y un cuantificador existencial), la definición sería circular, ${ }^{29}$ pero nada similar ocurre en el caso de las teorías aritméticas. Por eso, para evitar la acusación de arrogancia, en lugar de adoptar $\mathrm{PA}_{2}$ podríamos adoptar su condicionalización carnapiana. Si ésta es la situación, entonces las condiciones propuestas por HyW no son suficientes para afirmar que la versión condicional de $P A_{2}$ sea una definición implícita defectuosa. Tendríamos por ello dos teorías condicionalizadas, ninguna de las cuales es arrogante.

HyW no comparten este diagnóstico. ${ }^{30}$ Según explican, al ofrecer una definición implícita de \# por medio de $P H$ no sólo fijamos el valor semántico de una expresión aritmética, sino también adquirimos conocimientos a priori acerca de la aritmética. Estas dos cuestiones - la fijación del valor semántico y la adquisición de conocimientos- son, según ellos, separables. Una definición arrogante puede fijar apropiadamente el significado de la expresión sin por ello generar ningún tipo de conocimiento a priori sustantivo. De acuerdo con HyW, esto es lo que ocurre con $P A_{2}$. El problema de $P A_{2}$ no es su carácter no condicional, sino el hecho de que esta teoría no ofrece ningún tipo de información adicional a la ya introducida por su oración de Ramsey. Por lo tanto, el problema de $\mathrm{PA}_{2}$ sería que, si bien funciona como estipulación del significado de "cero", "sucesor", etc., no lo hace de un modo que genere alguna garantía a priori para creer en la verdad de lo estipulado.

A continuación HyW se preguntan si lo mismo puede decirse de $\mathrm{PH}$ y su respuesta es negativa. La razón que ofrecen es que no hay ninguna necesidad de estipular la verdad de $P H$ por medio de su oración de Ramsey, pero, para evitar la arrogancia, sí es necesario estipular la verdad de $P A_{2}$ por medio de su oración de Ramsey.

$\mathrm{Si}$ bien es cierto que no hay ninguna necesidad de postular la verdad de $P H$ por medio de su oración de Ramsey, no parece que eso sea relevante para evaluar si $\mathrm{PH}$ es arrogante. El argumento de HyW es que $P A_{2}$ es inaceptable porque no ofrece ningún tipo de información adicional sustantiva a la ya introducida por su oración de Ramsey, pero está claro que lo mismo puede decirse de $P H$, independientemente de si éste debe expresarse por medio de su oración de Ramsey o no.

${ }^{29}$ Aunque Horwich 2000 (p. 157) señala que el condicional en cuestión no debe ser el condicional material. Esto no soluciona el problema que discuto aquí por dos motivos. En primer lugar, se plantearía la misma acusación de circularidad para el nuevo condicional y, en segundo lugar, sigue en pie la acusación original para el cuantificador existencial.

${ }^{30}$ Véase, en particular, Hale y Wright 2009, pp. 468-473. 
De todas formas, considero que el argumento de $\mathrm{HyW}$ es confuso y más adelante ofreceré un argumento independiente en contra de la aptitud de la versión condicional de $P A_{2}$ como definición implícita. En particular, el escenario de paridad entre $F A$ y $P A_{2}$ sería diferente si el neologicista añadiera alguna condición extra que fuera capaz de clasificar como defectuosa a $P A_{2}$ en cualquiera de sus versiones. Una condición que HyW consideran pero que rápidamente rechazan es la unicidad. Lo que señalan a propósito de la unicidad es lo siguiente:

Primero, no hay un nivel absoluto de determinación, independiente de nuestros propósitos y del contexto particular, que nuestras explicaciones del significado deben alcanzar si han de ser adecuadas. Lo que debería contar como un constreñimiento insuficiente para un definiendum es relativo al contexto y a los propósitos de la definición. No es una objeción [correcta] a una definición implícita decir que ésta no logra distinguir entre varias interpretaciones más específicas de su definiendum si el contexto y los propósitos no exigen distinciones más finas. Segundo, no podemos pedir más determinación para los significados supuestamente fijados por definiciones implícitas que lo que pedimos para los significados en general. (Hale y Wright 2000, p. 292)

Sin embargo, dado que todo lo que necesitamos es ofrecer condiciones de identidad para el concepto o función que estamos definiendo, no parece demasiado pedir unicidad. Más aún, en este contexto las condiciones de identidad son extensionales, con lo cual el argumento de HyW para descartar la unicidad no parece del todo sostenible.

Ahora bien, necesitamos entender cuándo un operador está definido unívocamente. Nuel Belnap (1962), respondiendo a las conocidas críticas de Arthur Prior respecto de la conectiva tonk, sugiere que uno de los requisitos que las reglas inferenciales lógicas deben cumplir para caracterizar adecuadamente una conectiva es que la caractericen unívocamente. Por ejemplo, la conectiva plonk caracterizada exclusivamente por medio de la regla

$$
\psi / \varphi \text { plonk } \psi
$$

no cumple con el requisito de unicidad. Podemos introducir otra conectiva plink que definimos de manera idéntica a plonk pero que no expresa el mismo contenido inferencial. En cambio, las reglas que caracterizan las conectivas tradicionales sí cumplen con la unicidad. Es fácil mostrarlo si consideramos la conjunción. Sea " $\wedge$ " la conjunción tradicional y sea "\&" una conectiva gobernada exactamente por las mismas reglas que gobiernan la conjunción. Si podemos mostrar que $\varphi \wedge \psi$ y $\varphi \& \psi$ se

Diánoia, vol. LIX, no. 72 (mayo de 2014). 
implican mutuamente, habremos mostrado la unicidad de las reglas, $\mathrm{y}$ esto puede hacerse fácilmente.

Podemos mostrar también que algo similar sucede con "\#". Sea "\%" un operador definido implícitamente por medio del siguiente principio:

$$
P H^{\%} \quad \forall F \forall G(\%(F)=\%(G) \leftrightarrow F \approx G)
$$

donde "\%" es un operador del mismo tipo sintáctico que "\#". Es razonable suponer que \# define unívocamente el concepto "el número de..." si podemos probar que, para todo $F$ y todo $G, \# F=\# G$ y $\% F=\% G$ se implican mutuamente, lo cual parece cumplirse en la teoría que resulta de agregar $P H^{\%}$ a $F A$. Quizá pueda objetarse que esto no es suficiente para que la condición de unicidad sea satisfecha, pero es difícil ver qué otra cosa podría exigirse. Más aún, no parece sensato exigir más aquí de lo que exigimos en el caso de las conectivas lógicas, a saber, que el comportamiento inferencial de ambas expresiones sea el mismo.

La situación con la versión no arrogante de $P A_{2}$ es distinta. Aquí apelaremos a un ingenioso argumento ofrecido por Timothy Williamson (2003) en un contexto diferente. Lo que Williamson señala es que si condicionalizamos carnapianamente las definiciones implícitas para términos empíricos, éstas no cumplen con el requisito de unicidad de Belnap. El hecho de que los términos introducidos sean empíricos no cumple ningún papel en la prueba, de modo que el argumento es igualmente aplicable a términos aritméticos.

La idea de la prueba es la siguiente. Sea $\exists X \tau(X) \rightarrow \tau(H)$ la definición implícita cuya no unicidad se quiere probar. Si esta definición lograra caracterizar unívocamente el valor semántico de $H$, la siguiente fórmula sería una verdad lógica, donde $H_{1}$ y $H_{2}$ son variables de segundo orden:

$$
\text { Uni } \begin{aligned}
& \forall H_{1} \forall H_{2}\left(\left(\left(\exists X \tau(X) \rightarrow \tau\left(H_{1}\right)\right) \wedge\left(\exists X \tau(X) \rightarrow \tau\left(H_{2}\right)\right)\right)\right. \\
& \rightarrow\left.\forall x\left(H_{1} x \leftrightarrow H_{2} x\right)\right)
\end{aligned}
$$

Luego, lo que Williamson muestra es que si Uni es lógicamente verdadera, entonces $\exists X \tau(X)$ es lógicamente verdadera. Como sabemos que $\exists X \tau(X)$ no es lógicamente verdadera (ya que es la oración de Ramsey de una teoría no lógica), se inferirá que Uni no es lógicamente verdadera y que, por lo tanto, $\exists X \tau(X) \rightarrow \tau(H)$ no caracteriza unívocamente el significado de $H$.

[1] Supongamos que Uni es lógicamente verdadera. 
[2] Sabemos, por lógica proposicional, que $\neg \exists X \tau(X)$ implica lógicamente $\left(\exists X \tau(X) \rightarrow \tau\left(H_{1}\right)\right) \wedge\left(\exists X \tau(X) \rightarrow \tau\left(H_{2}\right)\right)$.

[3] Luego, si Uni es lógicamente verdadera, también será lógicamente verdadera $\forall H_{1} H_{2}\left(\neg \exists X \tau(X) \rightarrow \forall x\left(H_{1} x \leftrightarrow H_{2} x\right)\right)$.

[4] Y esta última fórmula es lógicamente equivalente a $\neg \exists X \tau(X) \rightarrow$ $\forall H_{1} \forall H_{2} \forall x\left(H_{1} x \leftrightarrow H_{2} x\right)$.

[5] Por lo tanto, si Uni es lógicamente verdadera, también será lógicamente verdadera $\neg \exists X \tau(X) \rightarrow \forall H_{1} \forall H_{2} \forall x\left(H_{1} x \leftrightarrow H_{2} x\right)$.

[6] Pero, suponiendo que siempre hay al menos un individuo en el dominio, la fórmula $\forall H_{1} \forall H_{2} \forall x\left(H_{1} x \leftrightarrow H_{2} x\right)$ es lógicamente falsa.

[7] En consecuencia, $\neg \exists X \tau(X)$ será lógicamente falsa.

[8] Con lo cual, $\exists X \tau(X)$ es lógicamente verdadera. Pero esto es falso, dado que es la oración de Ramsey de una teoría no lógica.

[9] De esto se sigue que Uni no es lógicamente verdadera.

[10] Luego, $\exists X \tau(X) \rightarrow \tau(H)$ no caracteriza unívocamente el significado de $H$.

Si $\tau$ es $P A_{2}$, este argumento muestra que la versión condicional de $P A_{2}$ no es una definición unívoca de los términos que define, a saber de "cero", "sucesor", "suma" y "multiplicación". Esta diferencia es, hasta donde puedo ver, lo único que le permite al neologicista mostrar la superioridad de $F A$ sobre la versión condicional de $P A_{2}$. Es decir, la no arrogancia no es suficiente para separar a $F A$ de la versión carnapiana de $P A_{2}$, pero la unicidad junto con la no arrogancia sí lo es.

Una réplica posible del defensor de $P A_{2}$ es que el neologicista también está obligado a condicionalizar carnapianamente su teoría porque sólo por medio de una condicionalización de este tipo puede evitarse la arrogancia. ${ }^{31}$ Desafortunadamente, esta réplica no parece llegar muy lejos.

En primer lugar, si bien creo que el neologicista no ha dado una explicación clara acerca de qué tipos de condicionalizaciones son suficientes

${ }^{31}$ Una complicación adicional es que si convertimos en una oración de Ramsey a $F A, \exists X \tau(X)$ será una fórmula de tercer orden, ya que la expresión que debe reemplazarse por la variable $X$ es \#, un símbolo de función de segundo orden. De modo que estamos suponiendo que el lenguaje ha sido enriquecido apropiadamente.

Diánoia, vol. LIX, no. 72 (mayo de 2014). 
para evitar la arrogancia, tampoco tenemos un argumento que nos indique que el tipo de condicional presente en $\mathrm{PH}$ no evita la arrogancia. Hasta que no dispongamos de una explicación más clara acerca de la no arrogancia, el defensor de $P A_{2}$ no podrá afirmar que $P H$, a pesar de su carácter condicional, no evita la arrogancia.

En segundo lugar, aunque se acepte que algún tipo de condicionalización carnapiana de $F A$ es deseable, el argumento de Williamson no se aplica a $F A$ porque su lógica subyacente es libre. En el paso [6] de la prueba de Williamson, para mostrar que $\neg \exists X \tau(X)$ es lógicamente falsa, se dice que la fórmula $\forall H_{1} \forall H_{2} \forall x\left(H_{1} x \leftrightarrow H_{2} x\right)$ es lógicamente falsa. No obstante, si hay modelos con el dominio vacío - y efectivamente los hay en la lógica libre subyacente que emplea el neologicista- ${ }^{32}$ la fórmula será contingente. Una manera de ver esto es notando que lo que esta fórmula expresa es que para cualesquiera dos propiedades se da que son, en términos extensionales, la misma propiedad. Si consideramos un modelo donde el dominio es vacío $\varnothing$, las variables de predicado tomarán sus valores del conjunto potencia del dominio, en este caso, $\{\varnothing\}$. Por lo tanto, en ese modelo la fórmula será trivialmente verdadera. Como hay una sola propiedad, se sigue que, para cualesquiera dos propiedades, son la misma propiedad. ${ }^{33}$ En cambio, si en la prueba de Williamson reemplazamos $\exists X \tau(X) \rightarrow \tau(H)$ por la condicionalización carnapiana de $P A_{2}$, el paso [6] se lleva a cabo normalmente, dado que la lógica subyacente de $P A_{2}$ no es libre. Por lo tanto, no hay razones para pensar que la condicionalizacion carnapiana de FA no ofrece una caracterización unívoca de \#.

\section{Observaciones finales}

He sugerido en primer lugar que las condiciones ofrecidas por HyW no son suficientes para mostrar que ciertas versiones de $\mathrm{PA}_{2}$ no son definiciones implícitas adecuadas de "cero", "sucesor", etc. En particular, la condición de no arrogancia no es suficiente para descartar ciertas versiones condicionales de $P A_{2}$. He argumentado además que el problema tiene una solución. El neologicista debe aceptar la unicidad entre las

\footnotetext{
${ }^{32}$ Una posible objeción es que la lógica no tiene por qué admitir dominios vacíos (es decir, podría ser libre sin ser inclusiva); pero obsérvese, de nuevo, que HyW admiten explícitamente la posibilidad de que el dominio sea vacío.

${ }^{33}$ En cambio, si hubiera al menos un objeto en el dominio la fórmula no sería lógicamente verdadera. Sea $\{a\}$ el dominio. Las variables de predicado toman como valores elementos del conjunto potencia del dominio, a saber, elementos de $\{\varnothing,\{a\}\}$. Luego, el valor de la variable $H_{1}$ puede ser $\varnothing$, y el de $H_{2}$ puede ser $\{a\}$.
} 
condiciones. En ese caso, la teoría $P A_{2}$ será arrogante en el sentido de HyW, mientras que la versión condicional de $P A_{2}$ no será unívoca.

Puede decirse, entonces, que el neologicista se enfrenta al siguiente dilema. O bien la unicidad no es una de las condiciones que las definiciones implícitas deben satisfacer para ser adecuadas, o bien sí lo es. En caso de que no lo sea (éste es el camino que eligen HyW), la no arrogancia debe ser suficiente (dejando de lado las consideraciones sobre la generalidad y la armonía) para descartar $P A_{2}$, en cualquiera de sus versiones. Sin embargo, he sugerido que la explicación de la no arrogancia que ofrecen HyW es insuficiente para determinar de manera general qué tipos de condicionales son arrogantes y qué tipos de condicionales no lo son. Esto hace que sea por lo menos difícil determinar rigurosamente si la versión condicional $P A_{2}$ es arrogante o no lo es. En caso de que la unicidad sea una de las condiciones (éste es el camino que, en mi opinión, los neologicistas deberían seguir), sí es posible descartar $P A_{2}$, dado que tenemos un argumento que muestra que la versión condicional más prometedora de $P A_{2}$ no logra ser una definición implícita unívoca.*

\section{BIBLIOGRAFÍA}

Belnap, Nuel, 1962, “Tonk, Plonk and Plink”, Analysis, vol. 22, no. 6, pp. 130134.

Bencivenga, Ermanno, 1986, "Free Logics", en Handbook of Philosophical Logic, ed. D.M. Gabbay y F. Guenthner, vol. 3, Alternatives to Classical Logic, Kluwer, Dordrecht, pp. 373-426.

Boghossian, Paul, 2008, Content and Justification, Oxford University Press, Nueva York.

_ 2003 , "Blind Reasoning", Proceedings of the Aristotelian Society, Supplementary Volume 77, no. 1, pp. 225-248.

Boghossian, Paul y Christopher Peacocke (comps.), 2000, New Essays on the A Priori, Clarendon Press, Oxford.

Boolos, George, 1998a, Logic, Logic, and Logic, Harvard University Press, Cambridge.

_ 1998 b, "Is Hume's Principle Analytic?", en Boolos 1998a, pp. 301-315.

Boolos, George, 1987, "The Consistency of Frege's Foundations of Arithmetic", en J. Thomson (comp.), On Being and Saying: Essays in Honor of Richard Cartwright, The MIT Press, Cambridge, Mass., pp. 3-20.

*Quiero agradecer a un evaluador anónimo de la revista Diánoia sus valiosas correcciones y sugerencias. Este artículo fue escrito con la ayuda financiera del Consejo Nacional de Investigaciones Científicas y Técnicas.

Diánoia, vol. LIX, no. 72 (mayo de 2014). 
Cook, Roy (comp.), 2007, The Arché Papers on the Mathematics of Abstraction, Springer, Dordrecht.

Frege, Gottlob, 1974, The Foundations of Arithmetic, 2a. ed. rev., trad. J.L. Austin, Basil Blackwell, Oxford.

Hale, Bob y Crispin Wright, 2011, "The Metaontology of Abstraction”, en David Chalmers et al. (comps.), Metametaphysics: New Essays on the Foundations of Ontology, Oxford University Press, Oxford, pp. 178-212.

— 2009, "Focus Restored: Comments on John MacFarlane", Synthese, vol. 170, no. 3 (ed. Øysten Linnebo), pp. 457-482.

— 2001, The Reason's Proper Study: Essays Towards a Neo-Fregean Philosophy of Mathematics, Clarendon Press, Oxford.

- 2000, "Implicit Definition and the A Priori", en Boghossian y Peacocke 2000, pp. 286-319.

Horwich, Paul, 2000, "Stipulation, Meaning, and Apriority", en Boghossian y Peacocke 2000, pp. 150-169.

MacFarlane, John, 2009, "Double Vision: Two Questions About the Neo-Fregean Program", Synthese, vol. 170, no. 3 (ed. Øysten Linnebo), pp. 443-456.

Nolt, John, 2010, "Free Logic", en Stanford Encyclopedia of Philosophy, The Metaphysics Research Lab, Stanford University, disponible en < http://plato. stanford.edu/entries/logic-free/ $>$.

Potter, Michael y Timothy Smiley, 2001, "Abstraction by Recarving", Proceedings of the Aristotelian Society, vol. 101, no. 3, pp. 327-338.

Priest, Graham, 2008, An Introduction to Non-Classical Logic, Cambridge University Press, Cambridge.

Prior, Arthur, "The Runabout Inference-Ticket", Analysis, vol. 21, 1960, pp. 3839.

Rumfitt, Ian, 2003, "Singular Terms and Arithmetical Logicism", Philosophical Books, vol. 44, no. 3, pp. 193-219.

Shapiro, Stewart, 1991, Foundations without Foundationalism. A Case for Second-Order Logic, Clarendon Press, Oxford.

Shapiro, Stewart y Alan Weir, 2007, “'Neo-Logicist' Logic Is Not Epistemically Innocent", en Cook 2007, pp. 119-146.

Smith, Peter, 2007, An Introduction to Gödel's Theorems, Cambridge University Press, Cambridge.

Williamson, Timothy, 2007, The Philosophy of Philosophy, Blackwell, Oxford.

— 2003, "Understanding and Inference", Proceedings of the Aristotelian Society, Supplementary Volume 77, no. 1, pp. 249-293.

Wright, Crispin, 2004, "Intuition, Entitlement, and the Epistemology of Logical Laws", Dialectica, vol. 58, no. 1, pp. 155-175.

— 2001a, The Reason's Proper Study: Essays Towards a Neo-Fregean Philosophy of Mathematics, Clarendon Press, Oxford.

— 2001b [1999], "Is Hume's Principle Analytic?", en Wright 2001a, pp. 307-334.

- 2001c [1997], "On the Philosophical Significance of Frege's Theorem", en Wright 2001a, pp. 272-306. 
Wright, Crispin, 1983, Frege's Conception of Numbers as Objects, Aberdeen University Press, Aberdeen.

Zalta, Edward, 2009, "Frege's Logic, Theorem, and Foundations for Arithmetic", en Stanford Encyclopedia of Philosophy, disponible en <http://plato. stanford.edu/entries/frege-logic/>.

Recibido el 18 de febrero de 2013; aceptado el 30 de enero de 2014.

Diánoia, vol. LIX, no. 72 (mayo de 2014). 